\title{
Comparison of Long and Short High-Intensity Interval Exercise Bouts on Running Performance, Physiological and Perceptual Responses
}

\section{(ㄷ)(1) (오 $\Theta$}

Authors

Sverre Andre Valstad ${ }^{1}$, Erna von Heimburg ${ }^{1}$, Boye Welde ${ }^{2}$, Roland van den Tillaar ${ }^{1}$

\section{Affiliations}

1 Nord University, Levanger, Sports Sciences and Physical Education, Levanger, Norway

2 UiT The Arctic University of Norway, School of Sports Sciences, Tromsø, Norway

Key words

accumulated oxygen uptake, aerobic exercise, postexercise metabolism, resting metabolic rate

received $\quad 27.06 .2017$

revised $\quad 02.10 .2017$

accepted 27.11.2017

\section{Bibliography}

DOI https://doi.org/10.1055/s-0043-124429

Sports Medicine International Open 2018; 2: E20-E27

(C) Georg Thieme Verlag KG Stuttgart · New York

ISSN 2367-1890

\section{Correspondence}

Prof. Roland van den Tillaar,

Nord University

Levanger, Sports Sciences and Physical Education

Odins veg 23

Levanger, 7603

\author{
Norway \\ Tel.: +47/976/62 913 \\ roland.v.tillaar@nord.no
}

ABSTRACT

This study compared the effects of long $(4 \times 4 \mathrm{~min})$ and short intervals $(4 \times 8 \times 20 s)$ of high-intensity interval exercise bouts (HIIT) on running performance, physiological and perceptual responses, and excess postexercise oxygen consumption (EPOC). Twelve healthy college students ( 8 men, 4 women; mean age $=22 \pm 2$ years) performed long (90-95\% of peak heart rate) and short intervals (maximal intensity) of high-intensity training (running on a non-motorized treadmill) with the same total duration on separate days. The total volume of consumed oxygen during recovery was the same in both cases $(P=0.21)$, whereas the short intervals of high-intensity training were performed at a faster mean running velocity $(3.5 \pm 0.18$ vs. $2.95 \pm 0.07 \mathrm{~m} / \mathrm{s}$ ) and at a lower $R P E_{\text {breath }}$ compared with the long intervals of high-intensity training. The blood lactate concentration also tended to be lower during the short intervals of high-intensity training, indicating that short-interval training was perceived to be easier than long-interval training, even though the cardiovascular and metabolic responses are similar. Furthermore, EPOC lasted significantly longer (83.4 \pm 3.2 vs. $61.3 \pm 27.9 \mathrm{~min}, \mathrm{P}=0.016)$ and tended to be higher $\left(8.02 \pm 4.22=v s .5 .70 \pm 3.75 \mathrm{~L} \mathrm{O}_{2}, P=0.053\right)$ after short intervals than after long intervals of training.

\section{Introduction}

High-intensity interval training (HIIT), in a variety of forms, is one of the most effective means of improving cardiorespiratory and metabolic functions and health in the general population, as well as the performance of athletes [8]. During strenuous physical training, the metabolic rate may increase exponentially, and both training intensity and duration exert major effects on metabolic responses that occur during and after the exercise [5, 21, 28]. This type of training improves the cardiometabolic characteristics of healthy and obese individuals, as well as those with type 2 diabetes, to a greater extent than continuous aerobic exercise of mod- erate intensity. Furthermore, HIIT effectively restores vascular function in patients suffering from heart disease [14, 20, 24, 30, 31].

In addition to increasing considerably during strenuous physical training, the elevated metabolic rate may persist for a prolonged period afterward. This phenomenon, known as excess postexercise oxygen consumption (EPOC), may exert a major impact on total energy consumption [5, 21, 28]. Training status, the intensity and duration of exercise, and dietary energy content all affect metabolic responses both during and following training $[5,21,28]$. The importance of EPOC is found in the increased oxygen uptake; it therefore burns through more calories, which may be of impor- 
tance for weight loss. Therefore, to be able to recommend different HIIT training regimes, energy consumption during and after different HIIT protocols have to be investigated.

It was found that exercise of higher intensity elevated postexercise oxygen consumption () to a greater extent and more persistently than exercises at a lower intensity [3, 6, 12, 21, 23]. For example, Laforgia et al. [22] found that $20 \times 1$-min bouts of high-intensity treadmill running interspersed with 2 -min rest periods ( $20 \mathrm{~min}$ at $105 \%$ of maximal oxygen uptake $\left[\dot{\mathrm{V}}_{2 \text { max }}\right]$ ) performed by eight male middle-distance runners resulted in an EPOC value twice as great as that following an equal amount of continuous work at a lower intensity ( $30 \mathrm{~min}$ at $70 \%$ of $\left.\dot{\mathrm{V}}_{2 \max }\right)$. Moreover, Dawson et al. [12] observed that the exercise of highest intensity (34 min at $65 \%$ of $\dot{\mathrm{V}} \mathrm{O}_{2 \max }$ ) performed by eight healthy young women produced a substantially greater EPOC value than other regimens ( $41 \mathrm{~min}$ at $55 \%$ of $\dot{\mathrm{V}} \mathrm{O}_{2 \max }$ and $49 \mathrm{~min}$ at $45 \%$ of $\dot{\mathrm{V}} \mathrm{O}_{2 \max }$ ) that involved the same overall level of work.

Even though EPOC is significantly altered when a session of aerobic exercise is divided into two bouts, few studies have focused on EPOC after HIIT. Kaminsky et al. [19] showed that when women performed 50 min of continuous running or two 25-min intervals, both at $70 \%$ of $\dot{\mathrm{V}}_{2 \max }$, the total EPOC values were $1.4 \mathrm{~L}$ and $\sim 3.1 \mathrm{~L}$, respectively. Moreover, Almuzaini et al. [1] reported that following 30 min of continuous cycling or two 15-min intervals (separated by $6 \mathrm{~h}$ ), both at $70 \%$ of $\dot{\mathrm{V}} \mathrm{O}_{2 \max }$, the total EPOC values were $5.3 \mathrm{~L}$ and $7.4 \mathrm{~L}$, respectively. When Larsen et al. [23] compared three different regimens of aerobic exercise (i.e., one 4-min bout, four 4-min bouts at $85-95 \%$ of maximal heart rate, and 47 min of continuous exercise at $70 \%$ of maximal heart rate), they obtained EPOC values of $1.3 \pm 1.1 \mathrm{~L}, 2.9 \pm 1.7 \mathrm{~L}$, and $1.4 \pm 1.1 \mathrm{~L}$, respectively. In the two former of these studies, it was indicated that a higher EPOC was found after performing high-intensity training in several smaller intervals with the same percentage of oxygen uptake as in continuous exercise.

However, the shortest regimens employed were 4-min [22] or 1 -min [21] intervals. Intervals shorter than 1 min may place even more stress on the body because of running velocity is higher. This in turn may lead to a greater EPOC than intervals at a lower running velocity lasting more than $1 \mathrm{~min}$. Long intervals (e. g., $4 \times 4 \mathrm{~min}$ ) are very popular as a training model, which has been extensively studied in different populations [18]. Short intervals of $8 \times 20$ s, called the Tabata interval, are also used in endurance training [29]. It is

- Table 1 Anthropometrical and physiological characteristics of the 12 study subjects.

\begin{tabular}{|l|c|c|c|}
\hline Variable & $\begin{array}{c}\text { Females } \\
(\mathbf{n = 4})\end{array}$ & $\begin{array}{c}\text { Males } \\
(\mathbf{n = 8}\end{array}$ & $\begin{array}{c}\text { All subjects } \\
(\mathbf{n = 1 2})\end{array}$ \\
\hline Age (years) & $22 \pm 1$ & $22 \pm 2$ & $22 \pm 2$ \\
\hline Height $(\mathrm{m})$ & $1.68 \pm 0.10$ & $1.80 \pm 0.05$ & $1.76 \pm 0.09$ \\
\hline Body mass $(\mathrm{kg})$ & $61.4 \pm 5.1$ & $74.3 \pm 4.2$ & $70.0 \pm 7.7$ \\
\hline $\mathrm{BMI}\left(\mathrm{kg} / \mathrm{m}^{2}\right)$ & $21.7 \pm 1.2$ & $22.9 \pm 0.8$ & $22.5 \pm 1.1$ \\
\hline $\mathrm{VO}_{2 \mathrm{max}}(\mathrm{mL} / \mathrm{kg} / \mathrm{min})$ & $48.6 \pm 6.2$ & $61.1 \pm 4.8$ & $56.9 \pm 8.0$ \\
\hline $\begin{array}{l}\text { Peak heart rate } \\
\text { (beat/min) }\end{array}$ & $189 \pm 5$ & $190 \pm 8$ & $190 \pm 7$ \\
\hline
\end{tabular}

Note. Values are means $\pm \mathrm{SD}$. $\mathrm{BMI}$, body mass index; $\mathrm{VO}_{2 \max }$, maximal oxygen uptake. important to highlight that HIIT is very popular because it is considered a time-efficient way to improve performance.

Therefore, the purpose of this study was to compare high-intensity short and long intervals with respect to physiological and perceptual responses during and after sessions in healthy subjects. It was hypothesized that (1) the physiological variables (oxygen uptake, lactate concentration and heart rate) and perceptual variables (rate of perceived exertion) would be equal during exercise and (2) the shorter intervals of high-intensity training would result in a more pronounced EPOC.

\section{Materials \& Methods}

\section{Participants}

Twelve healthy college students ( 8 men, 4 women), aged 20 to 25 years ( $\triangleright$ Table 1 ), who regularly trained in running, soccer, and handball for a minimum of three times a week volunteered to participate in the study. Subjects who met one or more of the following exclusion criteria were not eligible to participate in the study: injuries that prevented subjects from running at high intensity during the exercise sessions, known diseases associated with systemic and pulmonary circulation, reluctance to refrain from nicotine or alcohol consumption, and involvement in another study. The subjects' anthropometrical and physiological characteristics are shown in $>$ Table 1. The study was approved by the Norwegian Social Science Data Services and carried out in accordance with the current ethical standards in sports and exercise research and of the journal [16]. All subjects were fully acquainted with the nature of the study and informed of the experimental risks before signing a written consent form to participate. It was explicitly stated to the subjects that they could withdraw from the study at any point. The study was conducted in accordance with the Declaration of Helsinki.

\section{Experimental design}

To test the physiological and perceptual responses during and after short intervals and long intervals of high-intensity running on a curved, non-motorized treadmill, we used a balanced repeated measures crossover design. This approach entailed gathering data on the subjects' completion of the two training sessions on separate test days in randomized order. The long intervals of high-intensity training involved four 4-min intervals at 90 to $95 \%$ of peak heart rate $\left(\mathrm{HR}_{\text {peak }}\right)[17,32]$, and the short intervals comprised four sets of eight 20-s intervals at the highest possible intensity with a 10-s break after each interval [29]. In both cases, the subjects had a 3-min active recovery period between each set. Therefore the total duration (including breaks) was $25 \mathrm{~min}$ in both cases.

\section{Pre-testing measurements}

Four weeks before the main experiment, the subjects' maximal oxygen uptake $\left(\dot{\mathrm{V}}_{2 \text { max }}\right)$ was measured during running on a conventional motorized treadmill. The subjects also had a familiarization session on the non-motorized treadmill one week before. To determine the subjects $\dot{\mathrm{V}}_{2 \max }$, the subjects performed uphill running on a motorized treadmill (h/p/cosmos quasar, h/p/cosmos sports $\&$ medical gmbh, Nussdorf-Traunstein, Germany). After a 15-min warm-up, the participants ran for 5 to 6 min at a constant inclina- 
tion of $10.5 \%$, with an individual starting velocity and a gradual increase of $1 \mathrm{~km} / \mathrm{h}$ each minute. An inclination of $10.5 \%$ was chosen because all subjects were used to uphill running. The test was considered to be a maximal effort if two of the following three criteria were met: (a) a plateau in $\mathrm{V}_{2}$ with increasing intensity of exercise, (b) respiratory exchange ratio above 1.15 , and (c) blood lactate concentration exceeding $8 \mathrm{mmol} / \mathrm{L}$ [4].

The oxygen uptake was monitored continuously with the Oxycon Pro (Erich Jaeger, Viasys Healthcare, Hoechberg, Germany) in "breath-by-breath" mode, and the average of the three highest 10 -s consecutive measurements determined the $\dot{\mathrm{V}}_{2 \max }$. The heart rate was registered every $10 \mathrm{~s}$ with a Polar RS 400 (Polar Electro, Kempele, Finland), and the highest stable heart rate value during the last minute of the test was defined as the peak heart rate $\left(\mathrm{HR}_{\text {peak }}\right)$. The portable LT-1710 Lactate Pro ${ }^{\mathrm{TM}}$ analyzer (Arkray Factory Inc., KDK Corporation, Shiga, Japan) was used to measure the blood lactate concentration immediately after the test. This analyzer needs a sample of only $5 \mathrm{uL}$ of blood (taken from the fingertip).

A familiarization test was carried out on a non-motorized treadmill (Woodway Curve, model 1.5, Woodway, Waukesha, WI, USA) to see how the subjects responded to performing the interval sessions on this particular treadmill. The subjects first ran for $20 \mathrm{~min}$ at $70 \%$ of $\mathrm{HR}_{\text {peak. }}$. Thereafter, they performed one 4 -min interval from the long interval session and one set of $8 \times 20$-s intervals from the short interval session, interrupted by 3 min of active moderate walking between sessions.

\section{Experimental procedures}

For the experimental intervention, the subjects arrived at the laboratory at the same time of day $\pm 1 \mathrm{~h}$ on two separate test days following a 12-h overnight fast before both tests and with $48 \mathrm{~h}$ to one week between the tests. The subjects were instructed to refrain from performing vigorous activity for $48 \mathrm{~h}$, consuming alcohol for $36 \mathrm{~h}$, and consuming any caffeine or nicotine for $12 \mathrm{~h}$ prior to each test day [11]. After their height and weight measurements were recorded, and after ten minutes of rest, the subjects underwent a 30 -min measurement of resting metabolic rate. To perform this measurement, the subjects were hooked up to the Oxycon Pro open-air spirometry system set to operate in "breath-by-breath" mode. The subjects were then instructed to wear a mask connected to the open air spirometry system and to lie in a supine position on a mattress while resting their head on a pillow for maximal relaxation. Baseline oxygen uptake was calculated within a 5-min period (see Calculations). The heart rate at baseline was recorded as the heart rate value that was registered in parallel with the baseline $\dot{\mathrm{V}}_{2}$ value. The blood lactate concentration at baseline was measured immediately after the 30 -min resting metabolic rate measurement phase.

After the resting metabolic rate measurements were performed, the subjects warmed up by running at $70 \%$ of $\mathrm{HR}_{\text {peak }}$ for 10 min on the non-motorized treadmill. Thereafter, they performed one exercise session with short or long intervals. By using a non-motorized treadmill, the subjects could run at their individual highest possible intensity during the short-interval session. They could also increase and decrease the velocity by themselves and stay on the treadmill for the whole duration of the sessions, including moderate walking on the treadmill during the recovery period. The subjects wore the mask for the entire duration of the sessions and oxygen uptake and heart rate were measured continuously. After each set of short and long intervals, blood lactate concentration was measured and the subjects' ratings of perceived exertion (RPE) were registered. To measure the subjects' RPE of fatigue associated with non-linear physiological responses, i. e., blood lactate concentration, Borg RPE 0-10 [26] was used (RPE fatigue ). RPE 6-20 scale was used to measure the subjects' RPE of the pulmonary ventilation [9] after each set ( $\left.R P E_{\text {breath }}\right)$. The average running velocity on the treadmill (treadmill belt velocity) was measured after each interval and set by Curve 1.5 software (Woodway, Waukesha, WI, USA), and the total running distance covered over each training session was recorded.

Immediately after each interval session, the mask was removed so the subjects could drink a glass of water. Thereafter, within 2 min of completing the exercise session, the subjects were instructed to lie down on the mattress and were again hooked up to the open-air spirometry system [11]. The subjects then underwent a recovery period, which involved lying in a supine position until the $90-\mathrm{min}$ limit, during which the oxygen uptake and heart rate were measured. A 90-min limit was chosen based on the findings of Larsen et al. [23], who found the longest lasting increase in $\mathrm{O}_{2}$ uptake during recovery in their study to be $70.4 \pm 24.8 \mathrm{~min}$. After every $30 \mathrm{~min}$ of measurements, the subjects were permitted, for their own wellbeing, a 2-min break, during which the mask was removed. During the resting metabolic rate and postexercise measurements, each subject rested in a quiet room, completely shielded from external interference. All tests were performed indoors in a laboratory with good ventilation and well-controlled ambient conditions, at a room temperature maintained between $21^{\circ} \mathrm{C}$ and $24^{\circ} \mathrm{C}$.

\section{Calculations}

Steady-state conditions for resting metabolic rate were achieved within a 5 -min period with $\leq 10 \%$ coefficient of variation for $\dot{\mathrm{VO}}_{2}$ and carbon dioxide production $\left(\dot{\mathrm{V} C \mathrm{C}_{2}}\right)$ [11]. The lowest average value of five consecutive 1 -min measurements was used as the baseline $\dot{\mathrm{V}}_{2}$ value. In the recovery period after the training sessions, a subject was considered to have reached baseline again when the average of five consecutive 1-min EPOC measurements with $\leq 10 \%$ coefficient of variation for $\dot{\mathrm{V}} \mathrm{O}_{2}$ and $\dot{\mathrm{V}} \mathrm{CO}_{2}$ [11] corresponded to the baseline values. The accumulated $\mathrm{O}_{2}$ uptake during the two training sessions and during the recovery was calculated by multiplying the $\mathrm{O}_{2}$ uptake for each minute by the time in min used during or after the sessions. The EPOC was measured in liters and was calculated based on accumulated $\mathrm{O}_{2}$ uptake from the end of the training session until the 90 -min limit or until baseline values were reached. A subject was considered to have reached baseline again when the average of five consecutive 1-min EPOC measurements with $\leq 10 \%$ coefficient of variation for $\dot{\mathrm{V}} \mathrm{O}_{2}$ and $\dot{\mathrm{V}} \mathrm{CO}_{2}[11]$ corresponded to the baseline $\dot{\mathrm{VO}}_{2}$ values. If baseline was not reached during the recovery time, EPOC was measured for the whole 90-min recovery period. The EPOC values were calculated using the following formula: EPOC $(\mathrm{L})=$ accumulated $\dot{\mathrm{VO}}_{2}(\mathrm{~L})-\left(\dot{\mathrm{VO}}_{2}\right.$ baseline $(\mathrm{L} / \mathrm{min}) \times$ time to $\dot{\mathrm{V}}_{2}$ baseline $\left.(\mathrm{min})\right)$. The duration of 
- Table 2 Physiological parameters at baseline, as well as total distance covered and accumulated oxygen uptake during exercise, accumulated $\mathrm{O}_{2}$ uptake and EPOC during the recovery period for the two training sessions.

\begin{tabular}{|c|c|c|}
\hline Variable & Long interval & Short interval \\
\hline$\dot{\mathrm{V}} \mathrm{O}_{2}(\mathrm{~L} / \mathrm{min})$ at baseline & $0.27 \pm 0.04$ & $0.26 \pm 0.04$ \\
\hline $\begin{array}{l}\text { Heart rate (beat/min) at } \\
\text { baseline }\end{array}$ & $51 \pm 7$ & $50 \pm 6$ \\
\hline $\begin{array}{l}\text { Blood lactate concentration } \\
(\mathrm{mmol} / \mathrm{L}) \text { at baseline }\end{array}$ & $2.1 \pm 0.4$ & $2.0 \pm 0.5$ \\
\hline Total distance covered (m) & $3531 \pm 707$ & $3438 \pm 627$ \\
\hline Accumulated $\mathrm{O}_{2}$ uptake (L) & $65.37 \pm 15.95$ & $64.28 \pm 14.63$ \\
\hline Time to baseline $(\min )^{a}$ & $61.3 \pm 27.9$ & $83.4 \pm 3.2^{*}$ \\
\hline Accumulated $\mathrm{O}_{2}$ uptake $(\mathrm{L})^{\mathrm{b}}$ & $28.85 \pm 5.80$ & $29.97 \pm 6.25$ \\
\hline $\operatorname{EPOC}(\mathrm{L})^{\mathrm{c}}$ & $5.70 \pm 3.75$ & $8.02 \pm 4.22$ \\
\hline EPOC to EOC ratio & $8.7 \pm 4.9$ & $12.1 \pm 4.4^{*}$ \\
\hline \multicolumn{3}{|l|}{ Note. Values are means $\pm S D$} \\
\hline \multicolumn{3}{|c|}{$\begin{array}{l}\text { a Time to baseline or until } 90 \text { min if baseline was not reached. } \\
\text { b Accumulated } \mathrm{O}_{2} \text { uptake during the } 90 \text {-min recovery period. } \\
\text { c EPOC until baseline or until } 90 \text { min if baseline was not reached. }\end{array}$} \\
\hline
\end{tabular}

* Significant $(\mathrm{P}<0.05)$ difference between the two training sessions.

EPOC was considered to be the time after completion of exercise to the first minute of the 5-min average that established the baseline $[24,26]$. The ratio of EPOC to exercise oxygen consumption (EOC) was calculated in accordance with the method used by Bahr and Maehlum [2].

\section{Statistical analyses}

Data were checked for normality with the Shapiro-Wilk test. A oneway analysis of variance (ANOVA) with repeated measures was used to test for differences between subjects' responses at baseline and during the two different training regimens. Furthermore, the total running distance, accumulated oxygen uptake during short and long intervals of high-intensity training, and recovery time to return to baseline between the two sessions were also tested with a one-way ANOVA. To compare the subjects' physiological $\left(\mathrm{O}_{2}\right.$ uptake, heart rate, lactate concentration), performance (average running velocity of each set), and perceptual responses (RPE) during the long and short intervals of high-intensity training, we used a 2 (short vs. long intervals) $\times 4$ (sets 1-4) ANOVA with repeated measures. A 2 (short vs. long intervals) $\times 6$ (end of exercise, $6 \mathrm{~min}$, $10 \mathrm{~min}, 30 \mathrm{~min}, 60 \mathrm{~min}, 90 \mathrm{~min}$ ) ANOVA with repeated measures on both variables was used to test for differences in oxygen uptake and heart rate between the two training regimens during the recovery period. Post hoc comparisons with Holm-Bonferroni corrections were conducted to detect differences. All results are presented as mean \pm SD. In those instances where the sphericity assumption was violated, Greenhouse-Geisser adjustments of the $P$ values were reported. The criterion level for significance was set at $P<0.05$. The effect size was evaluated with $\eta^{2}$ (partial eta squared), where $0.01<\eta 2<0.06$ constitutes a small effect, $0.06<\eta 2<0.14$ constitutes a medium effect, and $\eta 2>0.14$ constitutes a large effect [10]. Statistical analysis was performed with IBM SPSS Statistics for Windows, version 21.0 (IBM Corp., Armonk, NY, USA).

\section{Results}

No significant differences were found at baseline for $\dot{\mathrm{V}} \mathrm{O}_{2}$, heart rate, and lactate concentration values before starting the two test protocols ( $F \leq 2.4, P \geq 0.174, \eta^{2} \geq 0.02$, $>$ Table 2$)$. In addition, no significant differences in total distance covered $(F=3.4, P=0.093$, $\eta^{2}=0.24$, $>$ Table 2$)$ and accumulated oxygen uptake $(F=1.1$, $\mathrm{P}=0.31, \mathrm{\eta}^{2=0.09},>$ Table 2 ) during the two training protocols were found.

All physiological, perceptual, and performance variables were affected during both interval sessions $\left(F \geq 10.0, P \leq 0.005, \eta^{2} \geq 0.48\right.$, - Fig. 1 and 2), and post hoc comparisons showed that the perceptual (RPE) and physiological (oxygen uptake, lactate concentration and heart rate) parameters increased significantly for every interval set under both conditions. Mean and peak oxygen uptake and heart rate during the entire training sessions (including breaks), as well as oxygen uptake and heart rate after the last interval set, did not differ between conditions $(0.66 \leq F \leq 1.58,0.14 \leq P \leq 0.53)$.

During the two different interval sessions, the mean running velocity was faster when the subjects performed the short intervals of high-intensity training compared with the long intervals ( $3.5 \pm 0.18$ vs. $2.95 \pm 0.07 \mathrm{~m} / \mathrm{s})$ of high-intensity training $(F=49.7$, $P<0.001, \eta^{2=0.82}$ ), whereas the opposite was found for the oxygen uptake and $R P E_{\text {breath }}$ - that is, these values were significantly higher after the long intervals of high-intensity training compared with the short intervals of high-intensity training $(F \geq 4.9, P \leq 0.048$, $\eta^{2} \geq 0.32$, > Fig. 1 and $>2$ ). In addition, there was a trend of higher lactate concentration after each long interval of high-intensity training compared with the short intervals of high-intensity training $\left(F=4.2, P=0.063, \eta^{2}=0.28\right)$. Post hoc comparison showed that the running velocity was faster in each set for the short intervals compared with the long intervals, whereas the RPE $E_{\text {breath }}$ was significantly higher for the long intervals after the second and third sets ( $\vee$ Fig. 1), and the oxygen uptake was significantly higher for the long intervals after the third and fourth sets ( $>$ Fig. 2). No other significant differences $\left(\mathrm{RPE}_{\text {fatigue }}\right.$ and heart rate) during the two interval sessions were found ( $F \leq 1.2, P \geq 0.293, \eta^{2} \geq 0.02$, $>$ Fig. 1 and 2 ). Within-group comparisons showed a significant increase in running velocity for short intervals from the first set to the second set and from the third set to the fourth set, whereas for long intervals, there was a significant increase in running velocity from the third set to the fourth set ( $\triangleright$ Fig. 1).

Only two of 12 subjects reached baseline values within $90 \mathrm{~min}$ after both the long- and short- interval sessions. An additional four subjects reached baseline values within 90 min after the long-interval session, but not after the short-interval session. One subject reached baseline values within 90 min after the short-interval session, but not after the long-interval session. The remaining five subjects did not reach baseline values within 90 min after either the short- or the long-interval session. Recovery followed the same pattern after both interval sessions; the oxygen uptake and heart rate decreased exponentially after exercise, with no significant differences in development between the two interval sessions $(F \leq 2.0$, $P \geq 0.17, \eta^{2} \leq 0.16$, $>$ Fig. 3$)$. The time to baseline was significantly shorter after the long-interval session compared with the shortinterval session ( $\triangleright$ Table 2). Furthermore, a trend of higher EPOC 

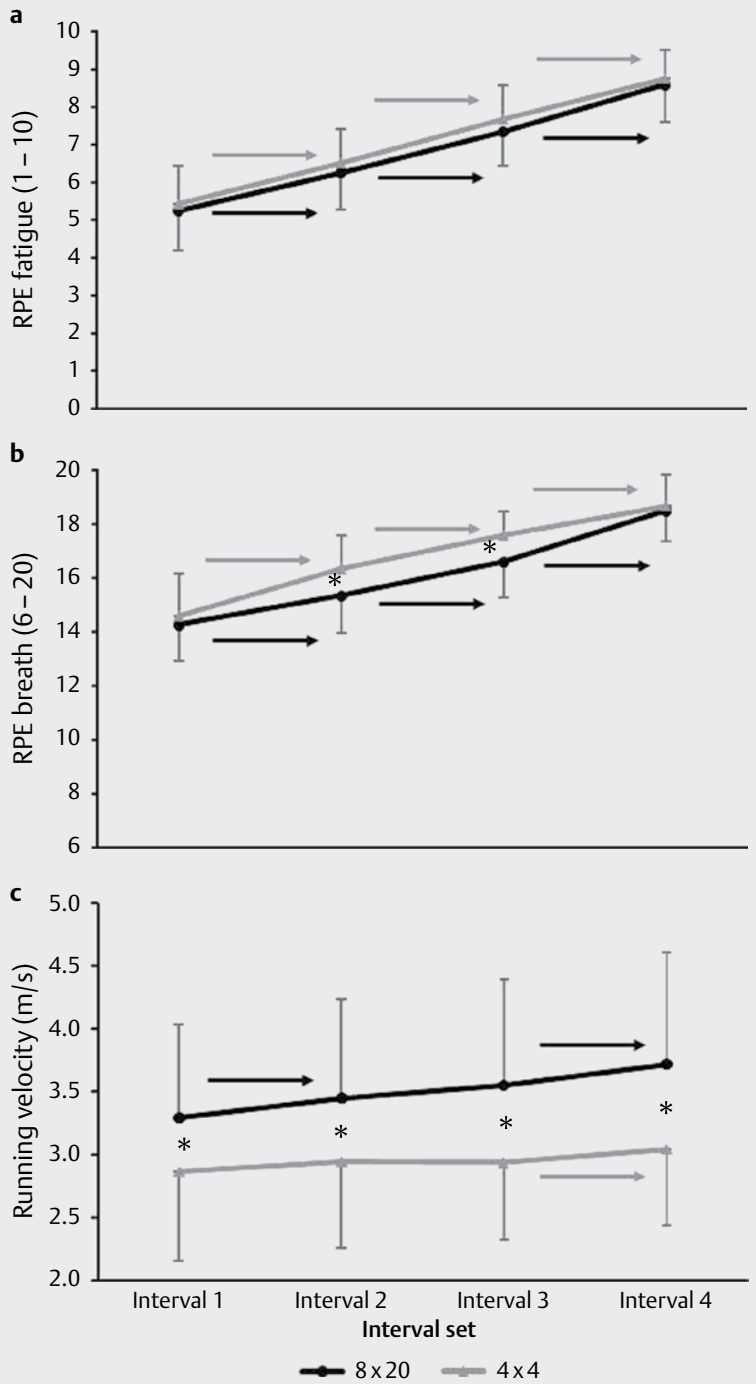

- Fig. 1 Rating of perceived exertion for muscle fatigue ( $\left(\mathrm{RE}_{\mathrm{fatigue}}\right)$ $\mathbf{a}$, rating of perceived exertion for breath ( $\left.\mathrm{RPE}_{\text {breath }}\right) \mathbf{b}$, and the average running velocity on the treadmill $\mathbf{c}$ and after each set during the short- and long-interval sessions. $\rightarrow$ indicates a significant increase from this value, $P<0.05$ level. * Significant $(P<0.05)$ difference between the short- and long-interval sessions at this set.

(in milliliters) until baseline, or until 90 min if baseline was not reached, was found for the short intervals compared with the long intervals $\left(F=4.7, P=0.053, \eta^{2}=0.30\right.$, $>$ Table 2$)$. There was no significant difference in accumulated $\mathrm{O}_{2}$ during the 90 -min recovery period between the groups $(F=1.7, P=0.21$, $>$ Table 2$)$. The ratio of EPOC to exercise oxygen consumption averaged $8.7 \pm 4.9 \%$ and $12.1 \pm 4.4 \%$ for the long intervals and the short intervals, respectively, and hence this ratio was higher for the short-interval protocol (mean difference $=3.4 \pm 5.3 \%, F=4.89, \mathrm{P}=0.049$ ).

\section{Discussion}

The main findings were that the ratio of EPOC to EOC was higher and EPOC duration was longer after the short intervals. Further-
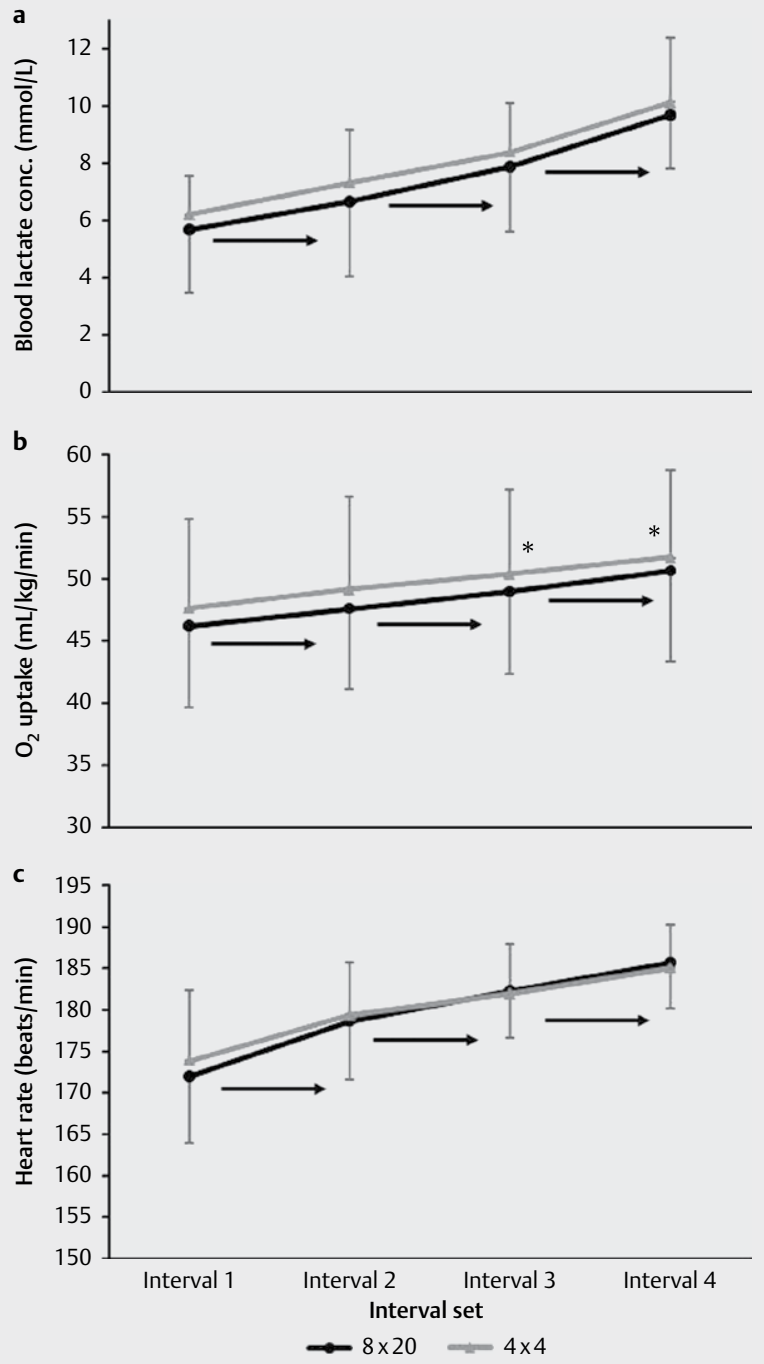

- Fig. 2 Oxygen uptake a, blood lactate concentration $\mathbf{b}$, and heart rate $\mathbf{c}$ after each set during the short- and long-interval sessions. $\rightarrow$ indicates a significant increase from this value, $\mathrm{P}<0.05$ level. * Significant $(P<0.05)$ difference between the short- and longinterval sessions at this set.

more, oxygen uptake and $\mathrm{RPE}_{\text {breath }}$ during the long intervals of highintensity training were higher than during the short intervals. There was no difference in total EPOC between the two experimental conditions.

No differences were found in oxygen uptake, heart rate, and blood lactate concentration between the two experimental conditions at baseline, suggesting that potential variance in baseline conditions did not affect the results. All physiological and perceptual variables were substantially elevated during the short- and longinterval training sessions. The similarity between protocols in accumulated oxygen uptake during training may indicate that the oxygen cost values were the same for the short- and long-interval training sessions. It is well known that energy expenditure increases with physical activity in proportion to the amount of work performed and that different interval training protocols with different 

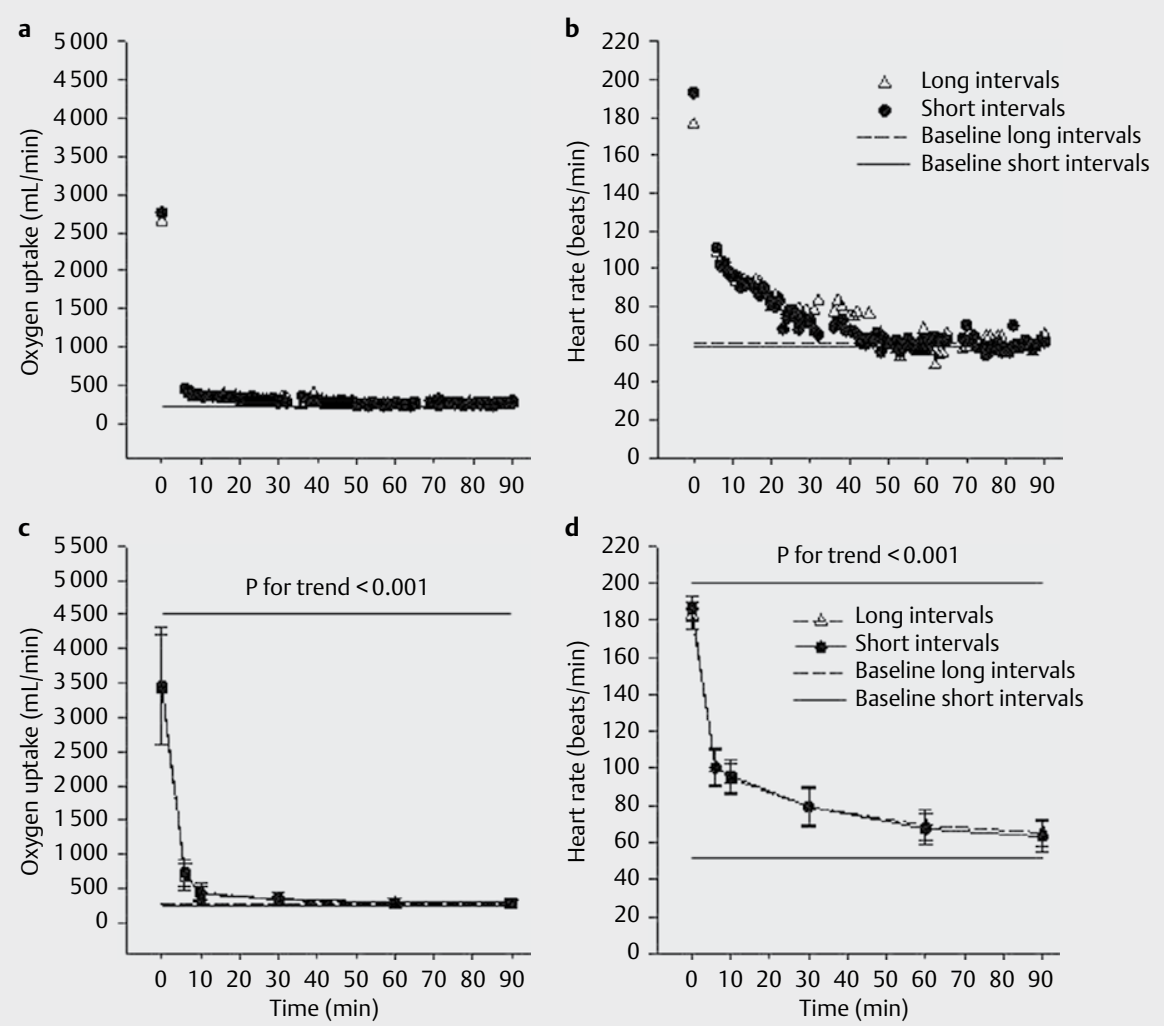

- Fig. 3 Decline in $\mathrm{O}_{2}$ uptake $\mathbf{a}, \mathbf{c}$ and heart rate $\mathbf{b}, \mathbf{d}$ from the end of the training to the end of the 90 -min recovery period. For all subjects pooled, $P<0.001$. All values significantly lower than first value $(P<0.001)$. Values after $6 \mathrm{~min}, 10 \mathrm{~min}, 30 \mathrm{~min}$, and 60 min were significantly lower than previous value $(P<0.01)$.

work-to-rest ratios may elicit different metabolic and cardiovascular responses [15]. The acute responses during the two experimental conditions in our study showed that both the short and long intervals of high-intensity training elicited similar substantial cardiovascular and metabolic responses.

In the present study, the total training duration was the same for the short and long intervals of high-intensity training, together with similar values for total distance covered ( $>$ Table 2 ) and accumulated oxygen uptake during exercise. This indicates that the cost of the exercise per se was the same in both conditions. The results, moreover, show that both interval protocols significantly increased acute metabolic and cardiovascular responses to a similar degree during exercise, even though the long-interval protocol had a total high-intensity work of 16 min (excluding breaks) and the short-interval protocol had a corresponding total high-intensity work of $10 \mathrm{~min}$ and $40 \mathrm{~s}$. Furthermore, the short intervals of high-intensity training were performed at a faster running velocity and at a lower $R P E_{\text {breath }}$ compared with the long intervals of high-intensity training. The blood lactate concentration also tended to be lower during the short intervals of high-intensity training, indicating, togeth-

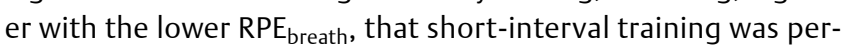
ceived to be easier to perform even though the cardiovascular and metabolic responses are similar to those in long-interval training. These findings may show the effect of training intensity, possibly highlighting the potentially greater efficiency of running at maxi- mal to supramaximal intensities of $\dot{\mathrm{V}}_{2 \max }$ compared with running at submaximal intensities.

After training, EPOC lasted longer and tended to be higher after short intervals of high-intensity training than after long intervals of high-intensity training. The total accumulated $\mathrm{O}_{2}$ uptake during recovery was the same in both cases; however, the ratio of EPOC to EOC was higher for short intervals of high-intensity training. It is well known that recovery from a bout of exercise is associated with an elevation in metabolism afterward. The findings of a $26 \%$ longer duration of EPOC after short intervals compared with long intervals of high-intensity exercise may reflect the fact that EPOC is sensitive to work intensity. In terms of oxygen uptake and heart rate during exercise, no differences between the two training protocols were found. However, the running velocity during the work intervals was $\approx 17 \%$ higher during the short-interval training protocol, which shows a greater exercise intensity during short intervals compared with long intervals of training. The total magnitude of EPOC did not differ between our interval protocols; however, short intervals of high-intensity training elicited a longer duration of EPOC compared with long intervals. This finding may be explained by the fact that only three subjects reached baseline values within $90 \mathrm{~min}$ following the short-interval protocol, whereas six subjects reached baseline values within the fixed 90-min time limit following the long-interval protocol. This longer duration of EPOC following the short intervals of very high-intensity running may suggest that 
these intervals elicited higher stress and greater impact on the longer-lasting slow component of the EPOC. Physiological mechanisms that could explain higher EPOC following the short intervals of higher-intensity running could be associated with a greater disturbance to exercising muscle mass during the short intervals, as suggested by Lyons et al. [25]. Moreover, Larsen et al. [23] found an average duration of EPOC of 70 min in male subjects with metabolic syndrome following $4 \times 4$ min HIIT, which was similar to our findings in healthy subjects. Nevertheless, our postexercise measurement period was clearly too short, given that only two subjects reached the baseline control value following both interval protocols. Because we stopped recording the postexercise measurements after $90 \mathrm{~min}$ and the subjects who had not yet reached baseline were assigned a value of $90 \mathrm{~min}$ for the duration of EPOC, we cannot tell the exact time needed to reach baseline in our study.

A difference in the ratio of EPOC to EOC, as introduced by Bahr and Maehlum [2], showed that EPOC averaged approximately $9 \%$ and $12 \%$ of EOC for, respectively, the long and short intervals of high-intensity training in our study. These findings may implicate a greater postexercise effect following short intervals compared with long intervals of high-intensity training. Sedlock et al. [27] found this ratio to be $9.7 \%$ following high-intensity exercise, whereas Kaminsky et al. [19] found it to be 1.3 to $2.8 \%$ following runs at $70 \%$ of peak oxygen uptake. These findings indicate that the interval protocols used in this study taxed the subjects' cardiovascular and metabolic system to a high degree, pointing out the significance of intensity with respect to postexercise effects.

A significant $\approx 0.55 \mathrm{~m} / \mathrm{s}$ faster running velocity during short intervals compared with long intervals of high-intensity training was found. It is interesting that the subjects ran faster during all four sets of the short intervals compared with the long intervals of highintensity training and that they were able to increase their running velocity over the entire short-interval session. Furthermore, the difference in running velocity between the two interval protocols also increased during the session. Generally, HIIT, in a variety of forms, is viewed as a time-efficient method for improving physical performance in athletes, but different formats of HIIT may still elicit different anaerobic energy contributions despite similar cardiorespiratory responses [7,8]. Similar metabolic and cardiovascular responses during exercise between short and long intervals of highintensity training were found. But it may be that the two interval protocols taxed the different energy systems to different degrees. Supposing that the short intervals of high-intensity training placed a higher demand on the splitting of the stored phosphagens than did the long intervals [13] owing to shorter work intervals and faster running velocity, it may be that the short-interval protocol is more sport-specific, at least for some groups of athletes. For example, track-and-field middle-distance runners, as well as athletes in team and racket sports, may benefit from the possible higher neuromuscular strain and the rapid shifts between intensive work periods and short breaks imposed by the short-interval protocol.

The total magnitude of EPOC in the current study may be underestimated because the rest intervals were not included in the calculation of total EPOC [21]. If a common baseline for the two experimental protocols had been chosen, the long intervals would have reached $\dot{\mathrm{VO}}_{2}$ at baseline at a later stage, and the short inter- vals would have reached $\dot{\mathrm{V}}_{2}$ at baseline at an earlier stage. As an example, in this study there was $\approx 15 \mathrm{~mL} \mathrm{O}_{2}$ in difference between the long intervals $(273.3 \mathrm{~mL} \mathrm{O}$ ) and the short intervals $(258.4 \mathrm{~mL}$ $\mathrm{O}_{2}$ ). If an average baseline had been chosen for resting metabolic rate for the long-interval protocol and the short-interval protocol, the average time to baseline would have been almost equal, with outcomes of $\approx 75 \mathrm{~min}$ for the long intervals and $\approx 74 \mathrm{~min}$ for the short intervals. If so, the levels of total EPOC would also have been more similar between the two interval protocols, with outcomes of $\approx 6,248 \mathrm{~mL} \mathrm{O}_{2}$ for the long intervals and $7,076 \mathrm{~mL} \mathrm{O}_{2}$ for the short intervals. Even if levels of EPOC become more similar in value between the two exercise protocols, there would still be a tendency for higher EPOC following the short intervals of high-intensity training.

\section{Conclusion}

This study showed that short intervals of high-intensity training contributed to faster running velocities than long intervals of highintensity training, although other physiological responses were the same. During recovery, the short-interval protocol elicited EPOC of a longer duration compared with the long-interval protocol, supporting previous evidence indicating that EPOC can be manipulated by changes in intensity of exercise. These findings show that even if both interval protocols were carried out with high intensity, the maximum intensity of the short intervals contributed to EPOC of longer duration and a tendency toward a greater total magnitude of EPOC. However, the ratio of EPOC to EOC shows that postexercise oxygen consumption contributes little to the daily energy expenditure and that it is the cumulative effect of the energy expenditure during the actual exercise that counts. Moreover, the faster running velocity during the short-interval protocol may make this protocol of greater relevance for some groups of athletes. A further practical application of the present study is that both the short and long interval produces a distinct postexercise elevation of metabolism, which may be relevant for weight loss.

\section{Acknowledgments}

This study was conducted without any funding from companies, manufacturers or outside organizations.

\section{Conflict of Interest}

The authors declare that they have no conflict of interest.

\section{References}

[1] Almuzaini KS, Potteiger JA, Green SB. Effects of split exercise sessions on excess postexercise oxygen consumption and resting metabolic rate. Can J Appl Physiol 1998; 23: 433-443

[2] Bahr R, Maehlum S. Excess post-exercise oxygen consumption. A short review. Acta Physiol Scand Suppl 1986; 556: 99-104

[3] Bahr R, Sejersted OM. Effect of intensity of exercise on excess postexercise $\mathrm{O} 2$ consumption. Metabolism 1991; 40: 836-841 
[4] Bassett DR Jr., Howley ET. Limiting factors for maximum oxygen uptake and determinants of endurance performance. Med Sci Sports Exerc 2000; 32: 70-84

[5] Borsheim E, Bahr R. Effect of exercise intensity, duration and mode on post-exercise oxygen consumption. Sports Med 2003; 33: 1037-1060

[6] Brockman L, Berg K, Latin R. Oxygen uptake during recovery from intense intermittent running and prolonged walking. J Sports Med Phys Fitness 1993; 33: 330-336

[7] Buchheit M, Laursen PB. High-intensity interval training, solutions to the programming puzzle. Part II: Anaerobic energy, neuromuscular load and practical applications. Sports Med 2013; 43: 927-954

[8] Buchheit M, Laursen PB. High-intensity interval training, solutions to the programming puzzle: Part I: cardiopulmonary emphasis. Sports Med 2013; 43: 313-338

[9] Chen M], Fan X, Moe ST. Criterion-related validity of the Borg ratings of perceived exertion scale in healthy individuals: a meta-analysis. J Sports Sci 2002; 20: 873-899

[10] Cohen J. Statistical power analysis for the behavioural sciences. 2nd ed.Hillsdale: Erlbaum; 1988

[11] Compher C, Frankenfield D, Keim N, Roth-Yousey L. Best practice methods to apply to measurement of resting metabolic rate in adults: a systematic review. J Am Diet Assoc 2006; 106: 881-903

[12] Dawson B, Straton S, Randall N. Oxygen consumption during recovery from prolonged submaximal cycling below the anaerobic threshold. J Sports Med Phys Fitness 1996; 36: 77-84

[13] Gastin PB. Energy system interaction and relative contribution during maximal exercise. Sports Med 2001; 31: 725-741

[14] Gibala MJ, McGee SL. Metabolic adaptations to short-term high-intensity interval training: A little pain for a lot of gain? Exerc Sport Sci Rev 2008; 36: 58-63

[15] Gosselin LE, Kozlowski KF, DeVinney-Boymel L, Hambridge C. Metabolic response of different high-intensity aerobic interval exercise protocols. J Strength Cond Res 2012; 26: 2866-2871

[16] Harriss D], Atkinson G. Ethical standards in sport and exercise science research: 2016 update. Int J Sports Med 2015; 36: 1121-1124

[17] Helgerud J, Hoydal K, Wang E, Karlsen T, Berg P, Bjerkaas M, Simonsen T, Helgesen C, Hjorth N, Bach R, Hoff J. Aerobic high-intensity intervals improve VO2max more than moderate training. Med Sci Sports Exerc 2007; 39: 665-671

[18] Helgerud J, Karlsen T, Kim WY, Hoydal KL, Stoylen A, Pedersen H, Brix L, Ringgaard S, Kvaerness J, Hoff J. Interval and strength training in CAD patients. Int J Sports Med 2011; 32: 54-59

[19] Kaminsky LA, Padjen S, LaHam-Saeger J. Effect of split exercise sessions on excess post-exercise oxygen consumption. $\mathrm{Br}$ J Sports Med 1990; 24: 95-98

[20] Kemi O], Wisloff U. High-intensity aerobic exercise training improves the heart in health and disease. J Cardiopulm Rehabil Prev 2010; 30: $2-11$
[21] LaForgia J, Withers RT, Gore C]. Effects of exercise intensity and duration on the excess post-exercise oxygen consumption. J Sports Sci 2006; 24: 1247-1264

[22] Laforgia J, Withers RT, Shipp N], Gore C]. Comparison of energy expenditure elevations after submaximal and supramaximal running. J Appl Physiol 1997; 82: 661-666

[23] Larsen I, Welde B, Martins C, Tjonna AE. High- and moderate-intensity aerobic exercise and excess post-exercise oxygen consumption in men with metabolic syndrome. Scand J Med Sci Sports 2014; 24 : e174-e179

[24] Little JP, Safdar A, Bishop D, Tarnopolsky MA, Gibala MJ. An acute bout of high-intensity interval training increases the nuclear abundance of PGC-1alpha and activates mitochondrial biogenesis in human skeletal muscle. Am J Physiol Regul Integr Comp Physiol 2011; 300: R1303R1310

[25] Lyons S, Richardson M, Bishop P, Smith J, Heath H, Giesen J. Excess post-exercise oxygen consumption in untrained men following exercise of equal energy expenditure: Comparisons of upper and lower body exercise. Diabetes Obes Metab 2007; 9: 889-894

[26] Noble B], Borg GA, Jacobs I, Ceci R, Kaiser P. A category-ratio perceived exertion scale: Relationship to blood and muscle lactates and heart rate. Med Sci Sports Exerc 1983; 15: 523-528

[27] Sedlock DA, Fissinger JA, Melby CL. Effect of exercise intensity and duration on postexercise energy expenditure. Med Sci Sports Exerc 1989; 21: 662-666

[28] Speakman JR, Selman C. Physical activity and resting metabolic rate. Proc Nutr Soc 2003; 62: 621-634

[29] Tabata I, Nishimura K, Kouzaki M, Hirai Y, Ogita F, Miyachi M, Yamamoto K. Effects of moderate-intensity endurance and high-intensity intermittent training on anaerobic capacity and VO2max. Med Sci Sports Exerc 1996; 28: 1327-1330

[30] Whyte LJ, Gill JM, Cathcart A]. Effect of 2 weeks of sprint interval training on health-related outcomes in sedentary overweight/obese men. Metabolism 2010; 59: 1421-1428

[31] Wisloff U, Ellingsen $\mathrm{O}$, Kemi O]. High-intensity interval training to maximize cardiac benefits of exercise training? Exerc Sport Sci Rev 2009; 37: 139-146

[32] Wisloff U, Stoylen A, Loennechen JP, Bruvold M, Rognmo O, Haram PM, Tjonna AE, Helgerud J, Slordahl SA, Lee SJ, Videm V, Bye A, Smith GL, Najjar SM, Ellingsen O, Skjaerpe T. Superior cardiovascular effect of aerobic interval training versus moderate continuous training in heart failure patients: A randomized study. Circulation 2007; 115: 3086-3094 